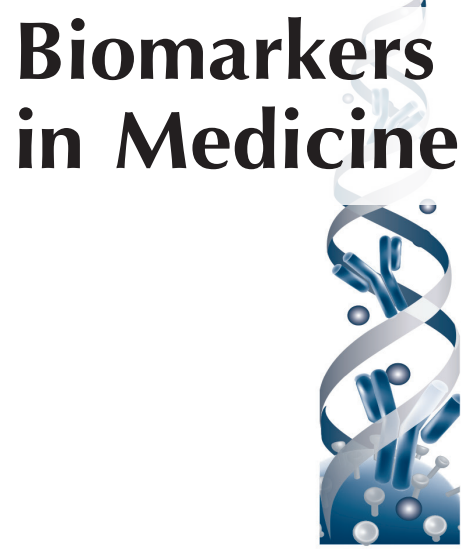

\title{
The National Biomarker Development Alliance accelerating the translation of biomarkers to the clinic
}

\begin{abstract}
"The end-to-end systems approach proposed by the National Biomarker Development Alliance, underpinned by broadly accepted standards, can dramatically reduce the number of candidate biomarkers eligible to move forward to development..."
\end{abstract}

Keywords: biomarker discovery $\bullet$ biomarker standards $\bullet$ biomarkers $\bullet$ biospecimens

- National Biomarker Development Alliance $\bullet$ precision medicine $\bullet$ translational research

\section{Biomarker development is rarely successful}

Realizing the much vaunted promise of precision (personalized) medicine is linked inextricably to the availability of robust and clinically relevant biomarkers. Given their importance in supporting more informed treatment decisions, enabling earlier diagnosis and driving the development of molecular based therapies, biomarkers arguably represent the greatest potential value in biomedicine today. In short, biomarkers are the holy grail' of precision medicine.

The BCR-ABL and HER-2 proteins are prototypic examples of the clinical utility of biomarkers in cancer that have transformed the classification of lymphocytic leukemia and HER-2 positive breast cancer, respectively, and driven more rational treatment decisions. Unfortunately, these remain rare examples. The potential of biomarkers to revolutionize the detection, diagnosis and clinical management of cancer remains largely unrealized, with fewer than 100 biomarkers approved by regulatory agencies as companion diagnostics for molecular profiling to guide treatment selection [1]. There is a staggering asymmetry between the several 100,000 publications describing candidate biomarkers and the mere fraction of $1 \%$ of these that ever progress to clinical practice [2]. The systematic failure in biomarker R\&D is further illustrated by the fact that the US FDA has approved less than one protein biomarker per year since the mid 1990s [3].
The burden of these failures, which come at incalculable cost in wasted investment, intellectual resources and patient samples, are incompatible with charting a reliable intellectual foundation for molecular medicine and improved patient care.

\section{Understanding why biomarkers fail}

Many of the root causes of failure in biomarker R\&D have been reviewed at length [3-8]. The most publicized biomarker failures, and certainly the most costly, are those that occur in advanced phase clinical trials. Regretfully, these failures are rarely investigated to identify why errors occurred and at what stage in the multiple steps involved in moving from discovery to clinical validation and regulatory approval. Far too often, these interdependent steps are disconnected and lack standardization. Errors that occur early in the R\&D cycle are often propagated undetected across these inadequately connected processes and undermine downstream validation making resulting failure inevitable.

Most biomarker discovery still takes place in government-funded university laboratories that are ill equipped to undertake the myriad procedures required for stringent biomarker profiling on the scale required to achieve the evidence needed to attract larger investment in clinical trials. Too often these discovery studies use biospecimens of dubious, unknown or unreported provenance, are statistically underpowered with insufficient sample numbers, and lack access to

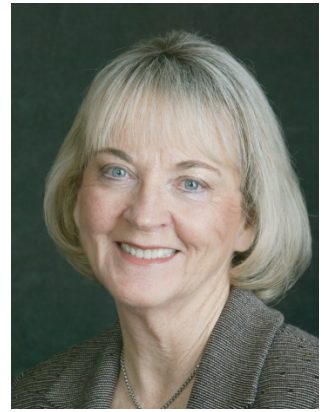

Anna D Barker

Author for correspondence: Complex Adaptive Systems, National Biomarker Development Alliance (NBDA), Arizona State University, SkySong, 1475 N. Scottsdale Rd, Suite 361, Scottsdale, AZ 85257, USA

Tel.: +1 4807270898

Fax: +1 4809658293

anna.barker@asu.edu

\section{Carolyn C Compton}

Complex Adaptive Systems, National Biomarker Development Alliance (NBDA), Arizona State University, SkySong, 1475 N. Scottsdale Rd, Suite 361, Scottsdale, AZ 85257, USA

\section{George Poste}

Complex Adaptive Systems, National Biomarker Development Alliance (NBDA), Arizona State University, SkySong, 1475 N. Scottsdale Rd, Suite 361, Scottsdale, AZ 85257, USA

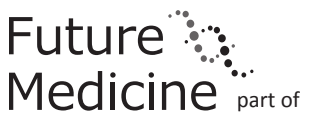


clinical data to demonstrate an unequivocal relationship between the biomarker(s) and clinical outcomes thereby limiting their perceived clinical utility and adoption.

\section{Failure begins in discovery}

While acknowledging that most flawed biomarker studies may involve multiple, cascading errors $[9,10]$, the penalty for mistakes in early discovery, especially bias in sample collection or lack of standards for collection and storage is manifest in late stage failure. The unacceptable consequences of not considering 'samples, standards and scale' when embarking on biomarker discovery has provoked calls for improved reproducibility in biomedical research, particularly in settings in which biomarker profiling has direct implications for treatment decisions. Sample selection, sources and quality must be relevant to the claimed clinical utility. Investigators ignore this key issue at their peril [11,12].

\section{Consequences of biomarker failure}

Collectively, the problems that plague biomarker R\&D often render the studies unable to answer the clinical question they set out to address [4]. In turn, this has created growing concern about the lack of reproducibility of many reported biomarker findings [9]. Most biomarkers fail when independent laboratories are unable to reproduce the original claims. In several very conspicuous cases, this has not occurred until very late in the development process despite earlier peerreviewed publications in authoritative scientific journals, formation of companies, hyped promotion in the lay press, and elevated patient expectations.

\section{“...biomarker R\&D is a modular, highly interdependent process that requires a systems-based, end-to-end approach to ensure seamless transfer of candidate markers across a series of modules from early discovery to clinical validation and their final regulatory approval."}

The lack of data sharing and forums for publication of negative results contributes significantly to this problem. Failed approaches may be repeated or compounded indefinitely, due to the near total lack of disclosure of raw data and failed efforts. Amgen recently reported that only six (11\%) of 53 seminal peer-reviewed published studies in drug discovery (a field completely dependent on robust biomarkers to identify therapeutic targets or toxicity) could be reproduced [10]. Sadly, little of practical value to the scientific community was derived from this effort because nondisclosure agreements blocked making the study details public. Similar studies by Bayer Healthcare reported higher, but still dismal reproducibility rates of $25 \%$ [13]. Reproducibility is a fundamental tenet of sound science. It is alarming that this problem exists at all, let alone so pervasively. Without robust reproducibility, biomarker development and clinical utility are all but impossible.

Even biomarkers that achieve clinical validation and FDA approval are endorsed as a practice standard and enjoy widespread clinical use and payer reimbursement may still suffer from undetected errors made earlier in the R\&D process. For example, following approval of the companion diagnostic HercepTest ${ }^{\circledR}$ by the FDA in 1998, widespread testing of breast cancer patients for HER 2 overexpression revealed false-positive and falsenegative rates of approximately $20 \%$ [14] Since this test is the key decision gateway to use of the life-saving but expensive targeted therapeutic Herceptin ${ }^{\circledR}$, the clinical and economic consequences of poor predictive test values are substantial. To correct the problem, the American Society of Clinical Oncology and the College of American Pathologists jointly developed and enforced standards in an approach that included test execution, operator proficiency and biospecimen handling [15]. This example illustrates an urgent need to identify where standards, applied throughout biomarker $R \& D$ could improve the translation of biomarkers into clinical application.

\section{Standards are the foundation of biomarker R\&D}

The newly launched National Biomarker Development Alliance (NBDA), represents a unique trans-sector alliance dedicated to solving this complex problem. The NBDA has spent nearly 2 years analyzing the biomarker discovery and development process using consultative think tanks involving all stakeholders (academics, clinicians, pharmaceutical and diagnostic companies, regulators, payers and patient advocacy groups). The consensus from this analysis was that biomarker $\mathrm{R} \& \mathrm{D}$ is a modular, highly interdependent process that requires a systems-based, end-to-end approach to ensure seamless transfer of candidate markers across a series of modules from early discovery to clinical validation and their final regulatory approval [16]. The NBDA analysis further determined that decision-making to successfully migrate a biomarker across the interdependent modules of early discovery, translational discovery, assay development and analytical validation, regulatory qualification and clinical validation requires definitive standards for each module and transition point.

It is not that standards do not exist. The literature is replete with claimed standards that are untested, ignored or applied sporadically in ways that fail to 
consider the multidimensional nature of the entire biomarker $\mathrm{R} \& \mathrm{D}$ process. As a result, there are no robust templates and protocols for predictable, standardsbased, end-to-end approaches for biomarker $R \& D$, no consistent guidelines, no best practices and inadequate decision criteria about whether to advance a biomarker to the next module in the overall process. This is a formula for disaster. Although the FDA has sought to raise the bar for biomarker regulatory submissions, they have been appropriately emphatic in indicating that the standards problem that plagues the biomarker 'ecosystem' must be addressed by its affected communities.

The NBDA has identified six strategic elements in biomarker R\&D that must be supported by appropriate standards, best practices and/or guidelines:

- The clinical question (correctly defined at the outset);

- Specimen provenance, quality and numbers;

- Experimental design and statistical robustness;

- Technology platform standards;

- Data and metadata quality;

- Analysis and analytics.

Failure of most biomarkers can be mapped back to these elements: small sample sizes; variable biospecimen quality and associated clinical data; inconsistent performance of measurement technologies and resulting test accuracy; flawed experimental design; poor data quality; faulty data analysis methods; and the omnipresent risk of bias associated with many of these parameters. Any of these issues, alone or in combination, or occurring at any phase in the biomarker $\mathrm{R} \& \mathrm{D}$ cycle can derail the entire process. Most important, a biomarker developed for clinical use must successfully inform a clinical decision that influences patient care. The prudent and logical planning process for biomarker R\&D would therefore be to work backwards from the end points of clinical need and regulatory requirements to define the best methods and standards needed to validate biomarkers to meet these performance specifications.

Flawed planning and implementation mistakes in any one of these strategic elements in the interconnected continuum from discovery to clinical validation will lead to failure. Integration of these strategic elements into a cogent end-to-end systems approach is obligate to guide biomarker development and as a vehicle to better learn from published biomarker failures and limit future repetition.

\section{A possible future}

A comprehensive systematic approach to biomarkers has not been undertaken or even seriously proposed perhaps due to the daunting multidisciplinary scope, scale and logistics. However, it can and must be confronted as an urgent imperative. There is a high level of frustration with the current level of poor productivity. Addressing this problem will require that the "silos" which charcterize the field give way to new collaborative, integrative approaches. The stakeholder communities (and funding agencies) must embrace a greater willingness to seek solutions via new collaborative trans-sector networks to mobilize the diverse expertise and substantial resources demanded by biomarker R\&D.

\section{"Biomarkers are key to achieving precision (personalized medicine) medicine."}

The NBDA was founded on the premise that the current dysfunctional and disjointed status of biomarker $\mathrm{R} \& \mathrm{D}$ can be re-engineered for success. It is a bold step towards the goal of advancement and coordination of standards and practices to achieve a seamless flow of meritorious biomarkers within and across the modules of the discovery and development pipeline. Only by adoption of a standards-based, end-to-end systems approach can we hope to redress the current situation which in any other field of research would be considered a catastrophic failure. Accomplishment of these audacious goals requires that the diverse stakeholders in the biomarker R\&D effort (academia, pharmaceutical and diagnostics companies, regulators, payers and patient advocates) work collaboratively to define and implement solutions

Biomarkers are key to achieving precision (personalized medicine) medicine. Although thousands of biomarkers are reported as 'discovered' each year, in areas such as oncology, less than one protein biomarkers per year have been approved by the US FDA since the early 1990s and fewer than 100 are routinely used in clinical practice. The root cause of most of these failures (and indeed the current national conversation on the lack of reproducibility in biomarker R\&D) is a glaring lack of accepted and broadly standards to inform every module and decision point of biomarker discovery and development. This problem is further compounded by the failure to view the biomarker $R \& D$ process as a highly interdependent end-to-end system. The newly launched National Biomarker Development Alliance represents a unique trans-sector approach to understand and solve these very complex problems.

The end-to-end systems approach proposed by the NBDA, underpinned by broadly accepted standards, can dramatically reduce the number of candidate bio- 
markers eligible to move forward to development; while simultaneously increasing the number of biomarkers with the credentials to pass scrutiny and survive the long and tortuous journey to the clinic.

\section{Financial \& competing interests disclosure}

$G$ Poste wishes to disclose assistance from the Board of Directors, Caris Life Sciences and Scientific Advisory Board, Syn-

\section{References}

1 Poste G. Bring on the biomarkers Nature 496, 156-157 (2011).

2 Kern SE. Why your new cancer biomarker may never work: recurrent patterns and remarkable diversity in biomarker failures. Cancer Res. 72, 6097-6101 (2012).

3 Ransohoff DF, Gourlay ML. Sources of bias in specimens for research about molecular markers for cancer. J. Clin. Oncol. 28, 698-704 (2010).

4 Hayes DF, Allen J, Compton C et al. Breaking a vicious cycle. Sci. Transl. Med. 5, 196 (2013).

5 Prinz F, Schlange T, Asadullah, K. Believe it or not: how much can we rely on published data on potential drug targets? Nat. Rev. Drug Discov. 10, 712 (2011).

6 Issaq, HJ, Waybright, TJ, Veenstra, TD. Cancer biomarker discovery: opportunities and pitfalls in analytical methods. Electrophoresis 32, 967-975 (2011).

7 Drucker E, Krapfenbauer K. Pitfalls and limitations in translation from biomarker discovery to clinical utility in predictive and personalised medicine. EPMA J. 4, 7 (2013).

8 Diamandis EP. The failure of protein cancer biomarkers to reach the clinic: why, and what can be done to address the problem? BMC Med. 10, 87 (2012). thetic Genomics. AD Barker wishes to disclose assistance from the Scientific Advisory Board, Caris Life Sciences. The authors have no other relevant affiliations or financial involvement with any organization or entity with a financial interest in or financial conflict with the subject matter or materials discussed in the manuscript apart from those disclosed.

No writing assistance was utilized in the production of this manuscript.

9 Mullard, A. Reliability of 'new drug target' claims called into question. Nat. Rev. Drug Discov. 10, 643-644 (2011).

10 Begley CG, Ellis LM. Drug development: raise standards for preclinical cancer research. Nature 483, 531-533 (2012).

11 Ransohoff DF. Lessons from controversy: ovarian cancer screening and serum proteomics. J. Natl Cancer Inst. 97, 315-319 (2005).

12 Cramer DW, Bast RC, Berg CD et al. Ovarian cancer biomarker performance in prostate, lung, colorectal, and ovarian cancer screening trial specimens. Cancer Prev. Res. 4, 365-374 (2011).

13 Pavlou, MP, Diamandis, EP, Blasutig IM. The long journey of cancer biomarkers from the bench to the clinic. Clin. Chem. 59, 147-157 (2012).

14 Winston JS, Ramanaryanan J, Levine E. HER-2/neu Evaluation in breast cancer are we there yet? Am. Soc. Clin. Pathol. 121, 533-549 (2004).

15 Wolff, AC, Hammond EH, Hicks DG et al. Recommendations for human epidermal growth factor receptor 2 testing in breast cancer: American society of clinical oncology/college of American pathologists clinical practice guideline update. J. Clin. Oncol. 31, 3997-4013 (2013).

16 NBDA Network. http://nbda-network.org/ 